\title{
WILSON FERMIONS AND THE TOPOLOGICAL CHARGE ON THE LATTICE
}

\author{
F. KARSCH \\ University of Illinois, Urbana, USA \\ E. SEILER ${ }^{1,2}$ \\ Department of Physics, Princeton University, Princeton, NJ 08544, USA \\ I.O. STAMATESCU \\ Institut für Theorie der Elementarteilchen, F.U. Berlin, F.R. Germany
}

Received 31 October 1985

\begin{abstract}
We investigate the realization on the lattice of the relation between the chiral properties of the fermions and the topological properties of the gauge field. A lattice definition of the topological charge density via the $\mathbf{U}(1)$ Adler-Bardeen anomaly is analysed with the help of given configurations of nontrivial topology and for 2-dimensional quenched QED.
\end{abstract}

\section{Introduction}

The Atiyah-Singer index theorem [1] and its generalization [2] express a deep connection between topological properties of the gauge field and the chiral properties of fermionic matter in Yang-Mills theories. In particular the topological charge density is equal to the U(1) Adler-Bardeen anomaly and this relation is in some sense equivalent to the index theorem [3].

The theorem, however, does not easily open itself to intuition. Also, in quantum field theory its meaning becomes somewhat obscure; in the continuum the gauge fields become too rough and in the infinite volume they fluctuate too much to ascribe topological quantum numbers to them in an easy way. On the other hand Witten and Veneziano [4] have made the idea of 't Hooft [5], that topology should solve the U(1) problem, more quantitative by expressing the mass of the $\eta^{\prime}$ in terms of the topological susceptibility which is a quantity characterizing the local fluctuations of the charge.

\footnotetext{
${ }^{1}$ Supported in part by NSF grant No. PHY 8116101-A02.

2 Permanent address: Max-Planck-Institut für Physik und Astrophysik, Werner-Heisenberg-Institut für Physik, Postfach 401212, D-8000 München, F.R. Germany.
} 
For these reasons it might be of interest to investigate the relation between anomalies and topology in a well-defined model. In perturbation theory the anomaly arises from the triangle graph only [6]. As the name "anomaly" indicates, it does not come out of the lagrangian in a straightforward way; it is produced by the fact that the functional integral has to be regularized and thereby loses some of its original naive symmetries. It can appear in different guises: either in the form of an effect of the jacobian under chiral transformations $[7,8]$ or in the form of an explicit chiral symmetry breaking term in the lagrangian of Wilson's lattice fermions [9]. As the cutoff is removed these two rather different looking things produce the same anomaly that is equal to the second Chern class in QCD.

In the following we will consider the lattice regularization and investigate more closely the definition of topological charge inherent in $[10,11]$ that keeps the relation to fermion properties intact (this connection seems to be lost with other definitions of the topological charge on the lattice [12]).

\section{The lattice chiral Ward identity for Wilson fermions}

The models we study are described by the following functional integral:

$$
\begin{gathered}
Z=\int\left[\mathrm{d} U_{n \mu}\right] \exp \left(-S_{\mathrm{YM}}\left(\left\{U_{n \mu}\right\}\right)\right) Z_{\mathrm{F}}\left(\left\{U_{n \mu}\right\}\right) \\
Z_{\mathrm{F}}\left(\left\{U_{n \mu}\right\}\right)=\int\left[\mathrm{d} \psi_{n}\right]\left[\mathrm{d} \bar{\psi}_{n}\right] \exp \left(-S_{\mathrm{F}}\left(\left\{U_{n \mu}\right\},\left\{\psi_{n}\right\},\left\{\bar{\psi}_{n}\right\}\right)\right),
\end{gathered}
$$

with $S_{\mathrm{YM}}$, e.g., the usual plaquette action

$$
S_{\mathrm{YM}}=-\frac{\beta}{N_{c}} \sum_{\mathrm{p}} \operatorname{Re} \operatorname{Tr}\left(U_{\partial p}\right)
$$

and $S_{\mathrm{F}}$ of the form

$$
\begin{aligned}
S_{\mathrm{F}}= & \sum_{n} \bar{\psi}_{n}\left(M+\frac{1}{2} \sum_{\mu}\left(\Gamma_{+}^{\mu}+\Gamma_{-}^{\mu}\right)\right) \psi_{n} \\
& -\frac{1}{2} \sum_{n, \mu=1}^{d} \bar{\psi}_{n}\left(\Gamma_{+}^{\mu} U_{n \mu} \psi_{n+\mu}+\Gamma_{-}^{\mu} U_{n-\mu, \mu}^{\dagger} \psi_{n-\mu}\right) .
\end{aligned}
$$

Here $[\mathrm{d} U]$ is the Harr measure, $\mathrm{d} \psi, \mathrm{d} \bar{\psi}$ denotes the Berezin integration [13] and we work on a euclidean lattice. Quite generally

$$
\Gamma_{ \pm}^{\mu}=r \mathrm{e}^{i \theta \gamma_{s}} \pm \gamma^{\mu},
$$


where $r=0$ corresponds to the so-called naive action. Notice that $r \neq 0$ only adds "irrelevant" terms to the naive action.

On the finite lattice $a \sim$ (cutoff) $)^{-1}>0$ ( $a$ is the lattice spacing) the chiral Ward identity is "normal". For the naive action $(r=0)$ which is chirally symmetric the axial current is conserved up to mass terms. This does not, however, express a conflict with perturbation theory because the naive action produces a proliferation of fermionic modes whose contributions to the anomaly agree with perturbation theory but cancel in the sum [14].

There are various possibilities of getting rid of this proliferation, some of them departing from the form given in (2.4) [15]. We consider the method expressed in eqs. (2.4), (2.5) with $r>0$ (so-called Wilson fermions) as the most transparent one and from now on we shall restrict our discussion to it.

In this case all Dirac components are still available at each lattice point and we can define local chiral transformations unambiguously; the fermionic determinant $Z_{\mathrm{F}}$ (eq. (2.2)) is invariant under them because they correspond to a linear transformation with determinant 1 of the variables in the Berezin integral. Therefore, as for the naive action, we obtain a "normal" chiral Ward identity which contains, however, an extra term coming from the term proportional to $r$ in eq. (2.4), which breaks chiral symmetry explicitly:

$$
\left\langle\partial_{\mu} J_{\mu S}(n)\right\rangle-2 M\left\langle J_{5}(n)\right\rangle+\langle X(n)\rangle=0,
$$

where

$$
\begin{aligned}
J_{\mu 5}(n)= & \frac{1}{2}: \bar{\psi}_{n} i \gamma_{5} \gamma^{\mu} U_{n \mu} \psi_{n+\mu}:+\frac{1}{2}: \bar{\psi}_{n+\mu} i \gamma_{5} \gamma^{\mu} U_{n \mu}^{\dagger} \psi_{n}:, \\
J_{5}(n)= & : \bar{\psi}_{n} i \gamma_{5} \psi_{n}: \\
X(n)= & \frac{1}{2} r \sum_{\mu}: \bar{\psi}_{n} \mathrm{e}^{i \theta \gamma_{5}} i \gamma_{5} U_{n \mu} \psi_{n+\mu}:+\frac{1}{2} r \sum_{\mu}: \bar{\psi}_{n+\mu} \mathrm{e}^{i \theta \gamma_{5}} i \gamma_{5} U_{n \mu}^{\dagger} \psi_{n}: \\
& +\frac{1}{2} r \sum_{\mu}: \bar{\psi}_{n-\mu} \mathrm{e}^{i \theta \gamma_{5}} i \gamma_{5} U_{n-\mu \mu} \psi_{n}: \\
& +\frac{1}{2} r \sum_{\mu}: \bar{\psi}_{n} \mathrm{e}^{i \theta \gamma_{S}} i \gamma_{5} U_{n-\mu \mu}^{\dagger} \psi_{n-\mu}:-2 \mathrm{~d} r: \bar{\psi}_{n} \mathrm{e}^{i \theta \gamma_{S} i \gamma_{5} \psi_{n}:}
\end{aligned}
$$

〈 > stands for the fermionic expectation value and the Wick dots mean subtraction of the free field $\left\{U_{n \mu}\right\}=\{1\}$ contribution. Then it can be shown that in the lowest order of perturbation theory [14] or quite generally for configurations where

$$
A_{n \mu} \equiv \frac{1}{i a}\left(U_{n \mu}-1\right)
$$


decreases fast enough at infinity $[10,16]$ :

$$
\lim _{a \rightarrow 0}\langle X(n)\rangle=\frac{-i}{16 \pi^{2}} F \tilde{F}(n)
$$

independently of $r>0, \theta$ (please note that in [10] a convention for $\theta$ is used that is shifted by $\pi$ compared to the standard one or, equivalently, $M$ is replaced by $-M$ ).

So the extra term $X$ leads to the usual anomaly in the continuum. There is a peculiar aspect to this result: the marginal quantity $X$ comes from the irrelevant supplementary term proportional to $r$ in the action. The explicit breaking of the chiral flavor symmetry also introduced by such a term in lattice QCD is expected to go away in the continuum limit (leaving behind a spontaneous breaking), whereas the chiral U(1) symmetry remains broken by the anomaly.

The limit in eq. (2.11) is understood for finite physical mass, i.e.

$$
M=m a \rightarrow 0, \quad m \text { fixed }
$$

and is not quite trivial. We can see that by looking at the normalization factor which is 1 in eq. (2.11).

For small but finite $a$ we have:

$$
\langle X(n)\rangle \cong-\frac{i}{16 \pi^{2}} I_{d}(m, a, r) F \tilde{F}(n)
$$

(here $d=2,4$ ) where

$$
I_{d}=-\frac{d a^{d}}{2 \pi^{d / 2}} \int_{0}^{2 \pi / a} \mathrm{~d}^{d} p \frac{\left(\Pi_{\mu} \cos a p_{\mu}\right) r\left(\sum_{\mu}\left(1-\cos a p_{\mu}\right)\right)\left(m a+r \sum_{\mu}\left(1-1 / \cos a p_{\mu}\right)\right)}{\left(\left(m a+r \sum_{\mu}\left(1-\cos a p_{\mu}\right)\right)^{2}+\sum_{\mu} \sin ^{2} a p_{\mu}\right)^{1+d / 2}}
$$

([10, 14, 16]; Yoshida, private communication). As a function of $r, a, I_{d}(d=2,4)$ behaves rather irregularly (G. Morchio, private communication; also $[10,14,16])$ :

$$
\lim _{\substack{a \rightarrow 0, m \text { fixed } \\ r \rightarrow 0, r>a^{1-\varepsilon}}} I_{d}(m, a, r)=1,
$$

whereas

$$
I_{d}(m, a, 0)=0 .
$$

For fixed $a, r I_{d}$ decreases fast with increasing $M$. In fig. 1 we show the behaviour of $I_{d}$ as a function of $M, r, L$ for lattices of various sizes $L$. We use $L$ also to fix the 

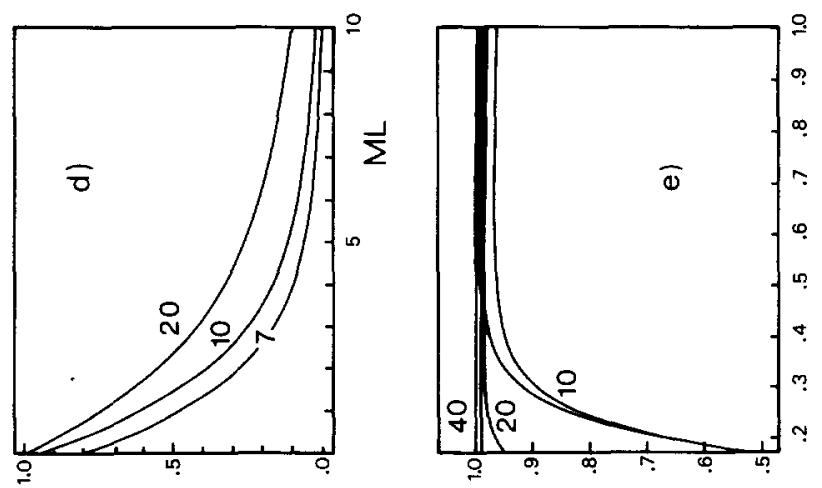

-i

$=\sqrt{11}$

$E=N \sum$

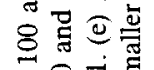

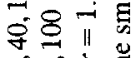

- 过守完

웡유

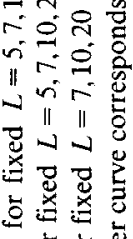

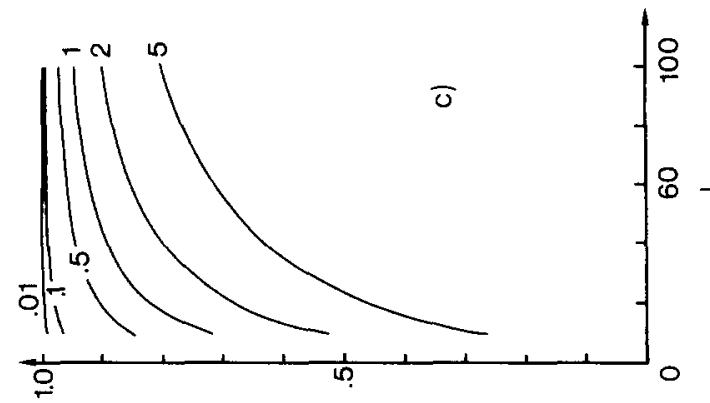

总总总高

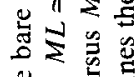

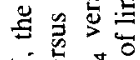

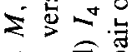

늘

过

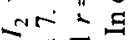

ล马 寻官

in in

in
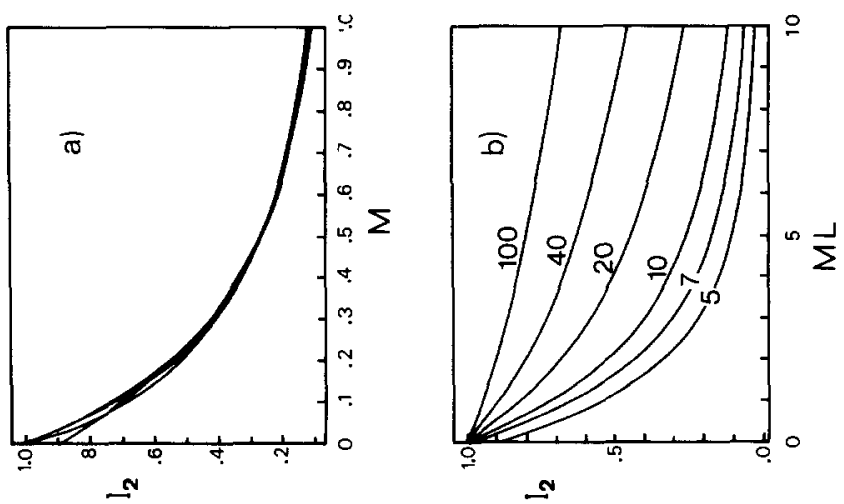

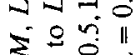

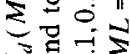

둥웅

농혀용

马용ㅇㅇ

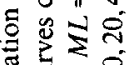

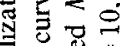

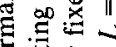

흥 뭉

के

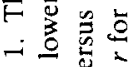

ㅇำ 
lattice spacing

$$
a \sim 1 / L
$$

and thus

$$
\begin{aligned}
& I_{d}(M, L, r)=-\frac{d 2^{d-1} \pi^{d / 2}}{L^{d}} \\
& \times \sum_{\left\{q_{\mu}=(\pi / L)\left(2 n_{\mu}+1\right)\right\}} \frac{\left(\Pi_{\mu} \cos q_{\mu}\right) r\left(\sum_{\mu}\left(1-\cos q_{\mu}\right)\right)\left(M+r \sum_{\mu}\left(1-1 / \cos q_{\mu}\right)\right)}{\left(\left(M+r \sum_{\mu}\left(1-\cos q_{\mu}\right)\right)^{2}+\sum_{\mu} \sin ^{2} q_{\mu}\right)^{1+d / 2}} .
\end{aligned}
$$

In fig. 1a we show $I_{2}$ as a function of $M$ for various $L$. We see that for fixed lattice parameter $M, I_{2}$ does not depend on $L$ for $L \geqslant 10$, i.e. the thermodynamic limit is reached quite early. Fig. 1b shows $I_{2}$ as function of $M L=M / a=m$ for various $L$, fig. 1c shows $I_{2}$ as function of $L$ for various $m$. One observes that for $m=M / a$ fixed, $a \rightarrow 0$ (i.e. $L \rightarrow \infty), I_{2} \rightarrow 1$ as expected. But this happens rather slowly, and we need a very small mass $M$ to obtain a sensible normalization factor $I_{d}$ for small lattices. Fig. $1 \mathrm{~d}$ is the correspondent of fig. $1 \mathrm{~b}$ for $d=4$ showing that the qualitative picture is preserved. Fig. 1e describes the $r$ dependence of $I_{2}$, for various (fixed) $m$, $L$ showing the "plateau" beginning at rather small $r$ values, $\sim 1 / L$. In the following we shall put $r=1$. These results were obtained for a given $\left\{U_{n \mu}\right\}$ configuration, i.e. an external Yang-Mills field. Results for the fully quantized theory have been obtained in low-order weak coupling perturbation theory and strong coupling expansion $[14,17,18]$.

\section{Lattice topological charge defined by way of the chiral Ward identity}

For the generalized Wilson action (2.4), (2.5) the term $X$, which is a candidate for the lattice topological charge density, depends on $M, r$ and $\theta$. Only in the continuum limit (2.11) this dependence goes away. In general we have [10]

$$
\int \prod_{n, \mu}\left[\mathrm{d} U_{n \mu}\right] \mathrm{e}^{-S_{\mathrm{YM}}} Z_{\mathrm{F}}(\theta)=\int \prod_{n, \mu}\left[\mathrm{d} U_{n \mu}\right] \mathrm{e}^{-S_{\mathrm{YM}}} \mathrm{e}^{-i \theta \tilde{X}(\theta)},
$$

with

$$
\begin{aligned}
\tilde{X}(\theta) & =\frac{i}{2 \theta} \int_{0}^{\theta} \mathrm{d} \theta^{\prime} X\left(\theta^{\prime}\right), \\
\frac{1}{2} i X(\theta) & =\frac{1}{i} \frac{\partial}{\partial \theta} \ln Z_{\mathrm{F}}(\theta)=\frac{1}{2} i \sum_{n}\left\langle X_{n}(\theta)\right\rangle .
\end{aligned}
$$


Since $Z_{\mathrm{F}}(\theta)$ is periodic in $\theta$ (see (2.4)) we can expand it in a Fourier series [10, 19]

$$
Z_{\mathrm{F}}(\theta)=\sum_{n} c_{n} \mathrm{e}^{i n \theta}
$$

Then $\frac{1}{2} i X(0)$ appears as some kind of average topological charge:

$$
\frac{1}{2} i X(0)=\left.\frac{1}{i} \frac{\partial}{\partial \theta} \ln Z_{\mathrm{F}}(\theta)\right|_{\theta=0}=\frac{\sum c_{n} n}{\sum c_{n}} .
$$

In the continuum limit we have

$$
\frac{1}{2} i X \underset{\substack{m \rightarrow 0 \\ m \text { fixed }}}{\longrightarrow} Q_{5}
$$

From now on let us take $r=1, \theta=0$. It is useful to parameterize the theory by the hopping parameter $k=(2 M+2 d)^{-1}$. $X_{n}$ has then a well-defined meaning on the finite lattice and converges to the topological charge density in the continuum limit. It can thus be considered as a candidate for the lattice topological charge density $[10,11]$. Recently it has been claimed that this definition does have no "perturbative tail" [20] which would make it really sensible and useful. In the following we look for the behavior of $X$ with $k$ to see if we can extract the information contained in (3.6) or (2.11) already at finite $a$. This would allow to observe the relation between chiral properties and topology directly in a well-defined model.

\section{Analytic properties of $X$}

\subsection{EXPECTED BEHAVIOR}

According to eqs. (2.9), (3.3) the quantity $X(k)$ is given by

$$
X(k) \equiv \sum_{n}\left\langle X_{n}(k)\right\rangle=\operatorname{Tr} \sum_{n, \mu} i \gamma_{5}\left(U_{n \mu}^{\dagger} G(n, n+\mu)+G(n+\mu, n) U_{n \mu}-2 G(n, n)\right)
$$

for $\theta=0, r=1$, where $G$ is the fermion propagator or, if we use the chiral Ward identity (2.6), by

$$
X(k)=\sum_{n} 2 M\left\langle J_{5 n}(k)\right\rangle, \quad\left\langle J_{5}\right\rangle=\operatorname{Tr}\left(i \gamma_{5} G\right)
$$

which can safely be used as long as $M>0$. Notice that $\gamma_{5} G$ is hermitic.

The hopping parameter expansion for $X=\sum X_{n}$ can be easily obtained and the coefficients of the Wilson loops can be calculated in the same way as for $Z_{F}$ itself 

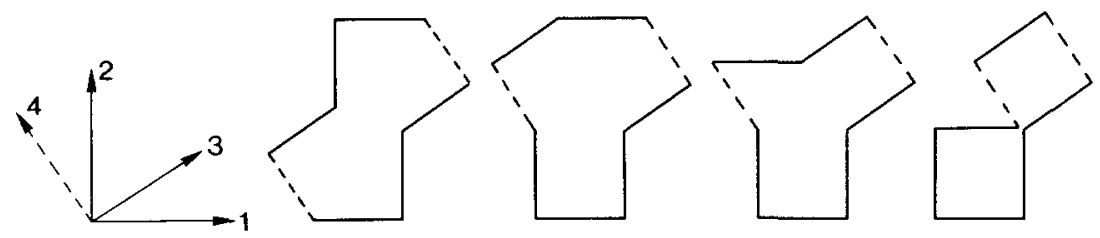

Fig. 2. Loops of order 8 contributing to $\frac{1}{2} i X(k)$. Each of the 4 topologically inequivalent classes contains $4 ! 2^{4}$ loops (up to translations), with the coefficients

$$
16 \varepsilon_{\mu_{1} \mu_{2} \ldots \mu_{8}}
$$

where $\mu_{i}= \pm \mu, \mu=1,2,3,4$ and $\varepsilon$ is the totally antisymmetric tensor

$$
\varepsilon_{1234-1-2-3-4}=1 \text {. }
$$

Allowed permutations are constrained by $(\mu \leftrightarrow \pm \nu) \rightleftharpoons(-\mu \leftrightarrow \mp \nu)$.

[21]: The result is (for $d=2,4$ ):

$$
X(k)=\frac{k-k_{0}}{k_{0}} \sum_{n=d}^{\infty}(2 d k)^{2 n} \sum_{\mathscr{L}_{2 n}} C_{\mathscr{L}_{2 n}} \operatorname{Tr} \mathscr{L}_{2 n},
$$

where $\mathscr{L}_{2 n}$ are Wilson loops of length $2 n$.

Already in lowest order there are many loops that occur in (4.3). For $d=4$ there are 4 types of loops of perimeter 8 which contribute each one together with all its permutations and rotations (see fig. 2). Clearly already at this order $X$ is much more complex than e.g. the definitions of topological charge given by Peskin [22] or Di Vecchia [23] (fig. $2 \mathrm{~d}$ or $2 \mathrm{a}$, respectively).

The hopping parameter expansion converges in a disc $|k| \leqslant\left|k_{\mathrm{c}}\right|$. For $k=k_{\mathrm{c}}$ the fermion determinant vanishes, presumably signalling the existence of a massless particle.

For free fermions the mass in units of $a^{-1}$ is

$$
M=\frac{1}{2 k}-d
$$

and thus

$$
k_{\mathrm{c}}=k_{0} \equiv \frac{1}{2 d} \text {. }
$$

(In a finite volume with antiperiodic boundary conditions this value is shifted by an amount $\mathrm{O}\left(L^{-1}\right)$ and actually moves off the real axis.)

In general $k_{\mathrm{c}}$ depends on the configuration $\left\{U_{n \mu}\right\}$ and satisfies $\left|k_{\mathrm{c}}\right| \geqslant k_{0}$ (cf. [17]. This fact can easily be proven by using the fact that both multiplication by $U_{n}$ and 
shift by a lattice unit correspond to unitary operators and hence have norm 1$)$. Since a zero of the determinant implies a pole in $\left\langle J_{5}\right\rangle$ also $X$ will have a pole at $k=k_{\mathrm{c}}$.

As an illustration consider a single plaquette, i.e. a $2 \times 2$ lattice with free boundary conditions. Let $\phi$ be the phase of the plaquette variable for gauge group $\mathrm{U}(1)$. Then the hopping parameter expansion can be summed to give

$$
\left\langle J_{5}(k)\right\rangle=\frac{32 k^{4} \sin \phi}{1+16 k^{8}+8 k^{4} \cos \phi} .
$$

This result has also been obtained by direct calculation using REDUCE. (4.6) has poles at

$$
k_{\mathrm{c}}= \pm \sqrt{\frac{1}{2}} \mathrm{e}^{i( \pm \pi+\phi) / 2}
$$

For $\phi= \pm \pi$ we have poles on the real axis (note that here $\left|k_{\mathrm{c}}(\phi)\right|=k_{\mathrm{c}}(0)$, unlike what is expected in general for more realistic lattices).

Now consider lattice configurations approaching continuum gauge fields with fixed nonzero topological charge.

According to the derivation in ref. [10] - see also sect. 2 - the limit is taken with $m=M a^{-1}$ fixed, $a \rightarrow 0$, i.e. with $k-k_{0} \rightarrow 0-$. At $k=k_{0}$, however, for finite $a X(k)$ as given by eqs. (4.1), (4.2) has a kinematical zero (note that $k=k_{0}$ means $M=0)$. What we expect to happen is indicated in fig. 3a: Only for $a=0$ does $X(k)$ go to a finite value at $k=k_{0}$, giving the topological charge of the configuration.

Such behavior could be produced by a pole in $k$ at a value $k_{\mathrm{c}}$ slightly above $k_{0}$ (possibly also a pair of poles with small imaginary parts) that approaches $k_{0}$ as $a \rightarrow 0$; this is expected anyhow because of the necessary zeroes of the determinant. Indeed, the smoother the configuration looks, considered on the unit lattice, i.e. the closer we are to the continuum, the more will $k_{\mathrm{c}}$ approach $k_{0}$

$$
k_{c}(a) \underset{a \rightarrow 0}{\longrightarrow} k_{0} \text {. }
$$

(4.8) follows also from the index theorem if we have a gauge field configuration of non-zero topological charge: The continuum Dirac operator for $m=0$ has at least one zero mode on the torus with antiperiodic boundary conditions, forcing the determinant to vanish at $k=k_{0}$ corresponding to $M=0$. (For zero topological charge (4.8) will not hold in general because the zeroes will be slightly off the real axis; the vanishing of $X$ at $k=k_{0}$ gives just the right answer $Q_{\text {top }}=0$.)

On the basis of this discussion and of the formulae (4.1) to (4.3) we expect the following behavior of $X(k)$ :

$$
X(k)=4 i d\left(k-k_{0}\right) \frac{k^{2 d}}{k^{2}-k_{c}^{2}(a)} f\left(k^{2}\right),
$$




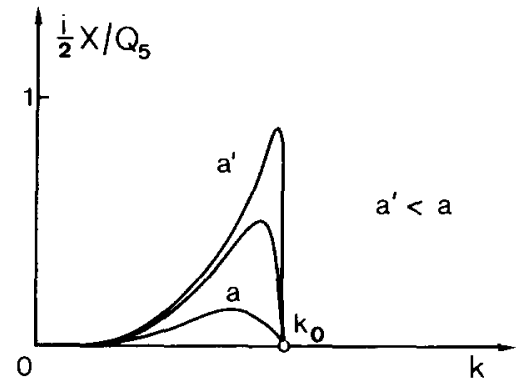

a)

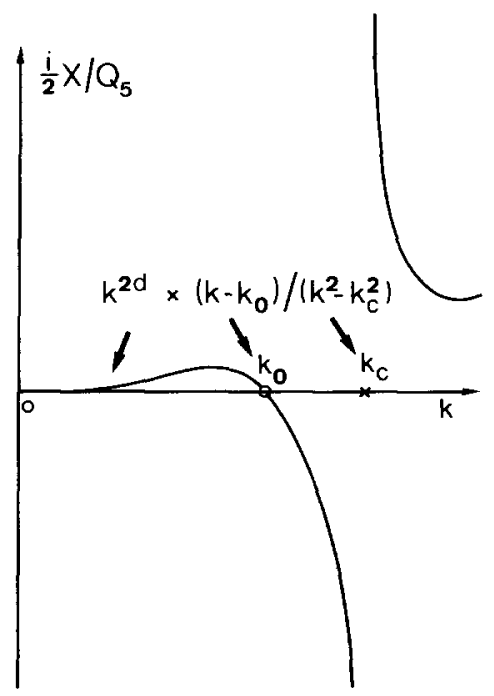

b)

Fig. 3. Expected behavior of $\frac{1}{2} i X(k)$. (a) The step-function-type of behaviour of $\frac{1}{2} i X(k)$ near $k_{0}$ for $a \rightarrow 0$. (b) Analytic structure implied by the ansatz eq. (4.9).

with a function $f\left(k^{2}\right)$ analytic in a domain containing both $k_{0}$ and $k_{\mathrm{c}}$ and depending only weakly on $a$ :

$$
f\left(k^{2}\right)=c_{0}+c_{1} k^{2}+\mathrm{O}\left(k^{4}\right)
$$

(note that $X\left(k-k_{0}\right)^{-1}$ has to be even in $k$ ). This is illustrated in fig. $3 \mathrm{~b}$.

For $a \rightarrow 0$ we would then have (using (4.8)):

$$
\lim _{k \rightarrow k_{0}-0} \lim _{k_{\mathrm{c}} \rightarrow k_{0}} \frac{1}{2} i X=-d k_{0}^{2 d-1} f\left(k_{0}^{2}\right) \equiv Q_{X}
$$

if we suppress the $a$ dependence of $f$ and regard $k_{\mathrm{c}}$ and $k$ as independent variables in (4.9). 
$Q_{X}$ should give the topological charge $Q_{5}$ of the configuration. In other words, the information about the topological charge is contained in the residue of the pole at $k=k_{\mathrm{c}}$ of $X(k)$.

Remark. In trying to implement this program one is sometimes faced with the following complication: There are additional pairs of zeroes and poles at or close to real values $k>k_{\mathrm{c}}$; this phenomenon is seen at higher topological charge. These additional structures do not affect in principle the preceding discussion; they stay separated from $k_{\mathrm{c}}$ as we approach the continuum and $k_{\mathrm{c}}$ approaches $k_{0}$. But they affect the speed of the approach to the continuum limit and sometimes reduce the effectiveness of the extrapolation formula (4.11).

\subsection{RESULTS FOR SIMPLE CONFIGURATIONS}

Periodic smooth Yang-Mills configurations with nontrivial topology can easily be constructed by taking, for instance, for $\mathrm{SU}(2)$ in 4 dimensions:

$$
\begin{aligned}
& U_{n, \hat{1}}=\exp \left(-2 \pi i \sigma_{3} n_{2} / L_{2}\right), \\
& U_{n, \hat{3}}=\exp \left(-2 \pi i \sigma_{3} n_{4} / L_{4}\right),
\end{aligned}
$$

where $\left\{n_{\mu}\right\},\left\{L_{\mu}\right\}(\mu=1,2,3,4)$ are the position vectors and the sides of the lattice respectively, and all other $U$ 's are 1 . Then

$$
\begin{aligned}
q_{5} & =\frac{1}{32 \pi^{2}} \varepsilon_{\mu \nu \rho \sigma} \operatorname{Tr} F_{\mu \nu} F_{\rho \sigma}=\frac{2}{L_{2} L_{4}}, \\
Q_{5} & =2 L_{1} L_{3} .
\end{aligned}
$$

We should find this number using our lattice definition for large $L_{\mu}$ (small $a$ ).

Similarly for $\mathrm{U}(1)$ in $d=2$ we take

$$
\begin{aligned}
& U_{n, \hat{1}}=\exp \left(2 \pi \operatorname{in}_{2} / L_{2}\right), \\
& U_{n, \hat{2}}=1 .
\end{aligned}
$$

This leads to

$$
\begin{aligned}
q_{5} & =\frac{1}{2 \pi} F_{12}=1 / L_{2}, \\
Q_{5} & =L_{1} .
\end{aligned}
$$

Because in $d=2$ there is no Bianchi identity the plaquettes in pure $\mathrm{QED}_{2}$ can be 
chosen independently and we can take an apparently less smooth configuration like

$$
U_{\left(n_{1}=1, n_{2}\right), \hat{1}}=\exp \left(2 \pi \operatorname{in}_{2} / L_{2}\right) \text {, }
$$

with all other $U$ 's equal to 1 , leading to a smaller topological charge

$$
Q_{5}=1
$$

(coming only from 1 column).

Intermediate values for $Q_{5}$ between 1 and $L_{1}$ can be obtained by considering configurations with a corresponding number of columns of links of the form eq. (4.16), for $n_{1}=1, \ldots, Q_{5}$.

Then for $L_{1}=L_{2}=L$ not too large we can calculate $J_{5}$ and $X$ explicitly as functions of $k$. Typical results are given in fig. $4 \mathrm{a}, \mathrm{b}$ showing complete agreement with the discussion in subsect. 4.1. To fit with the formulae (4.9)-(4.11) we take

$$
\frac{1}{2} i X(k)=2\left(k-k_{0}\right) \frac{k^{4}}{k_{0}^{3}}\left(k^{2}-k_{\mathrm{c}}^{2}\right)^{-2} Q_{X},
$$

with only two parameters $k_{\mathrm{c}}$ and $Q_{X}$ (remember $k_{0}=1 / 2 d=0.25$ ). The fit parameters are given in fig. $4 \mathrm{c}$ and table 1 and they obey roughly the relations

$$
\begin{aligned}
k_{\mathrm{c}} & \cong k_{0}+a_{1} / L, \\
Q_{X} & \cong Q_{5}\left(1-a_{2} / L\right),
\end{aligned}
$$

with $a_{1,2}$ smaller for configurations with smaller $Q_{5}$. Conversely we can look at the quantity

$$
\tilde{Q}_{X}(k) \equiv \frac{i}{2} \frac{k-k_{\mathrm{c}}}{k-k_{0}} X(k), \quad \tilde{Q}_{S} \equiv \tilde{Q}_{X}\left(k=\frac{1}{2}\left(k_{0}+k_{\mathrm{c}}\right)\right) .
$$

It is seen in fig. $4 \mathrm{a}, \mathrm{b}$ that $\tilde{Q}_{X}(k)$ is quite flat in the region $k_{0} \leqq k \leqq k_{\mathrm{c}}$ and stays near $Q_{5}$. No pole or other structure is seen for $Q_{5}=0$ (e.g., 2 opposite columns).

Thus the information about the continuum limit is indeed contained in the residue of $X(k)$ as conjectured in subsect. 4.1.

One might ask how this behavior relates to the derivation in ref. [10] and sect. 2. In fig. $4 \mathrm{~d}$ we show $\frac{1}{2} i X(k)$ just below $k_{0}$ : we see the behavior expected from fig. 3a, but the maximum stays well below $Q_{5}$ and even below $Q_{5} \times I_{d}\left(k_{\max }\right)$, indicating that the correction contained in $I_{d}$ is not sufficient and much larger lattices are needed for good agreement. Indeed, using the fit (4.19) in (4.18) we get for $k=k_{0}-\varepsilon$ :

$$
\frac{1}{2} i X\left(k_{0}-\varepsilon\right) \cong Q_{5}\left(1-\frac{a_{1}+8 a_{2}}{8 L}-\frac{3}{4} \varepsilon-\frac{a_{1}}{L \varepsilon}\right),
$$

indicating that one has to take $L^{-1} \ll \varepsilon \ll 1$. 
TABLE 1

Fit parameters (according to eq. (4.9)) and other quantities of interest for various smooth, 2-dimensional, abelian configurations

\begin{tabular}{|c|c|c|c|c|c|c|}
\hline \multicolumn{3}{|c|}{ Configuration } & \multirow[b]{2}{*}{$k_{\mathrm{c}}$} & \multicolumn{3}{|c|}{$\tilde{Q}_{X} / Q_{5}( \pm 1 \%)$} \\
\hline Lattice & $\mathrm{BC}$ & $Q_{5}$ & & at $k_{0}$ & at $k_{\mathrm{c}}$ & at $\frac{1}{2}\left(k_{0}+k_{\mathrm{c}}\right)$ \\
\hline $5 \times 5$ & $a p$ & 1 & 0.309214 & 0.7 & 1.52 & 1.09 \\
\hline \multirow[t]{6}{*}{$r=1$} & & $2^{+}$ & 0.33205 & 0.63 & 0.71 & 1.08 \\
\hline & & $3^{+}$ & 0.33467 & 0.56 & 0.53 & 0.91 \\
\hline & & 5 & 0.366517 & 0.55 & 1.64 & 1.05 \\
\hline & $p$ & 1 & 0.274546 & 0.71 & 1.25 & 0.96 \\
\hline & & 2 & 0.283752 & 0.44 & 0.67 & 0.57 \\
\hline & & 5 & 0.33793 & 0.5 & 1.35 & 0.87 \\
\hline $5 \times 5$ & $a p$ & 1 & 0.347463 & 0.74 & 1.55 & 1.13 \\
\hline$r=0.9^{\dagger \dagger}$ & & $2^{\dagger}$ & 0.372191 & 0.67 & 0.71 & 1.11 \\
\hline $6 \times 6$ & $a p$ & 1 & 0.288816 & 0.75 & 1.33 & 1.02 \\
\hline \multirow[t]{2}{*}{$r=1$} & & 2 & 0.308 & 0.7 & 1.4 & 1.0 \\
\hline & & 6 & 0.334671 & 0.59 & 1.4 & 0.97 \\
\hline $7 \times 7$ & $a p$ & 1 & 0.277585 & 0.79 & 1.23 & 1.0 \\
\hline \multirow[t]{2}{*}{$r=1$} & & 2 & 0.293882 & 0.73 & 1.33 & 1.01 \\
\hline & & 7 & 0.317475 & 0.62 & 1.31 & 0.94 \\
\hline $8 \times 8$ & $a p$ & 1 & 0.2707 & 0.82 & 1.18 & 1.0 \\
\hline \multirow[t]{2}{*}{$r=1$} & & 2 & 0.2848 & 0.76 & 1.27 & 1.0 \\
\hline & & 8 & 0.3066 & 0.65 & 1.26 & 0.93 \\
\hline $9 \times 9$ & $a p$ & 1 & 0.2661 & 0.85 & 1.16 & 1.0 \\
\hline \multirow{2}{*}{$r=1$} & & 2 & 0.2785 & 0.79 & 1.24 & 1.0 \\
\hline & & 9 & 0.2991 & 0.67 & 1.21 & 0.93 \\
\hline $10 \times 10$ & $a p$ & 1 & 0.263 & 0.86 & 1.11 & 0.98 \\
\hline \multirow[t]{3}{*}{$r=1$} & & -2 & 0.2738 & 0.81 & 1.17 & 1.0 \\
\hline & & $3^{\dagger}$ & 0.2755 & 0.69 & 0.82 & 0.82 \\
\hline & & -10 & 0.2934 & 0.69 & 1.15 & 0.93 \\
\hline $11 \times 11$ & $a p$ & 1 & 0.2606 & 0.88 & 1.1 & 0.98 \\
\hline \multirow[t]{2}{*}{$r=1$} & & 2 & 0.2703 & 0.83 & 1.16 & 1.0 \\
\hline & & 11 & 0.289 & 0.71 & 1.15 & 0.95 \\
\hline $12 \times 12$ & $a p$ & 1 & 0.2591 & 0.9 & 1.07 & 0.99 \\
\hline \multirow[t]{2}{*}{$r=1$} & & 2 & 0.2675 & 0.84 & 1.11 & 1.0 \\
\hline & & 12 & 0.2854 & 0.73 & 1.13 & 0.93 \\
\hline $13 \times 13$ & $a p$ & 1 & 0.2575 & 0.9 & 1.1 & 0.99 \\
\hline$r=1$ & & 2 & 0.2653 & 0.86 & 1.17 & 1.0 \\
\hline $14 \times 14$ & $a p$ & 1 & 0.2566 & 0.93 & 1.07 & 1.0 \\
\hline$r=1$ & & 2 & 0.2635 & 0.87 & 1.14 & 1.0 \\
\hline
\end{tabular}

${ }^{\dagger}$ Second pair of zero - pole present.

${ }^{\dagger+}$ Notice that here $k_{0}=0.27778$. 

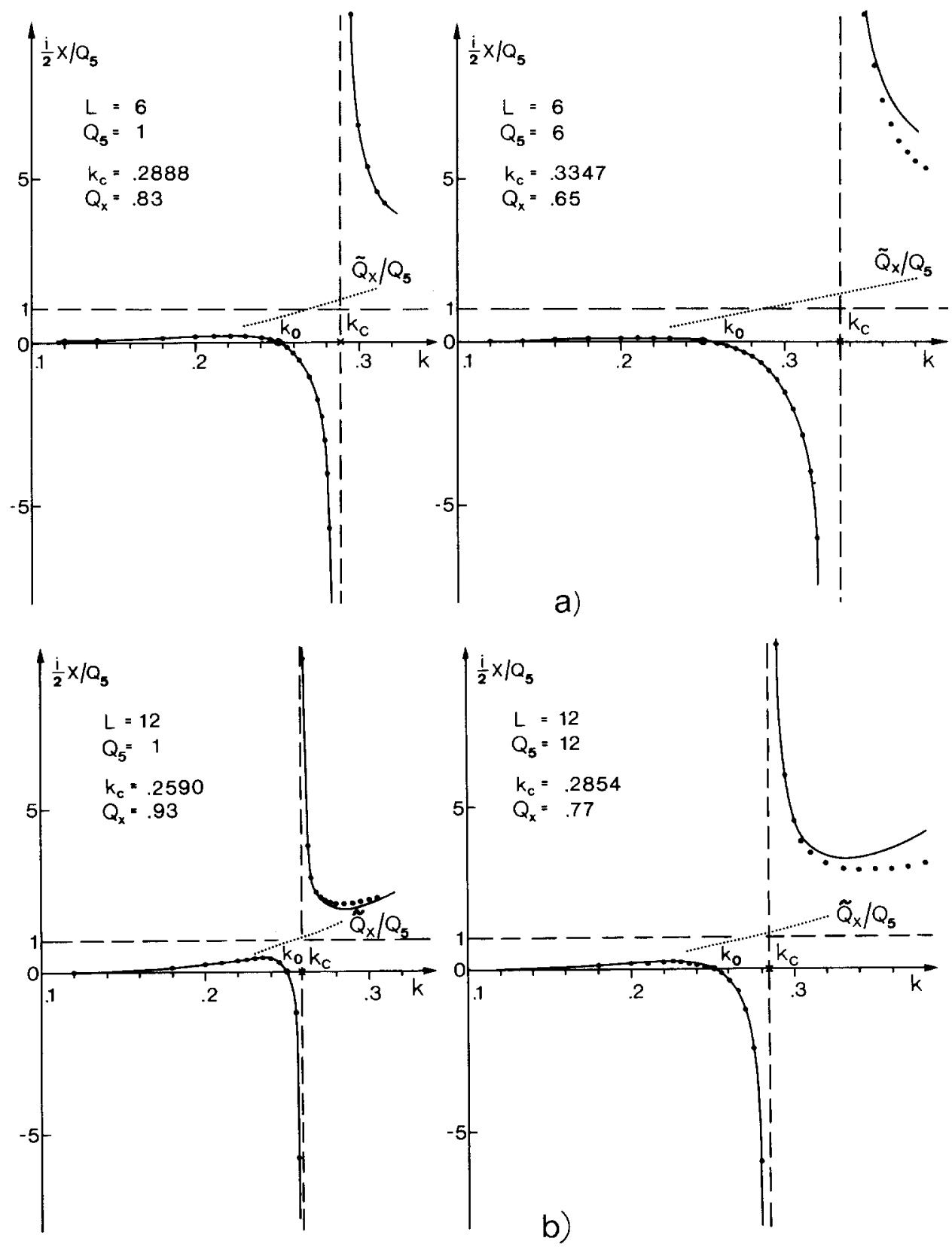

Fig. 4. $\frac{1}{2} i X(k)$ measured on 2-dimensional, abelian configurations with nontrivial topology. Here $Q_{5}=$ $(1 / 2 \pi) \sum_{\text {plaq }} F_{\mu \nu}, L$ is the lattice size and $r=1$. (a), (b) $\frac{1}{2} i X(k)$ calculated (dots), $\frac{1}{2} i X(k)$ fitted with eq. (4.9) (line) and $\tilde{Q}_{X}(k)=\left(k-k_{\mathrm{c}}\right) /\left(k-k_{0}\right) \frac{1}{2} i X(k)$ (dotted line) versus $k$ for various configurations. (c) The fit parameters $k_{\mathrm{c}}$ and $Q_{X}$ and the topological charge extracted as $\tilde{Q}_{5}=\tilde{Q}_{X}\left(k=\frac{1}{2}\left(k_{0}+k_{\mathrm{c}}\right)\right)$. (d) Indication of step-function-type of behaviour at $k_{0}-0$. 


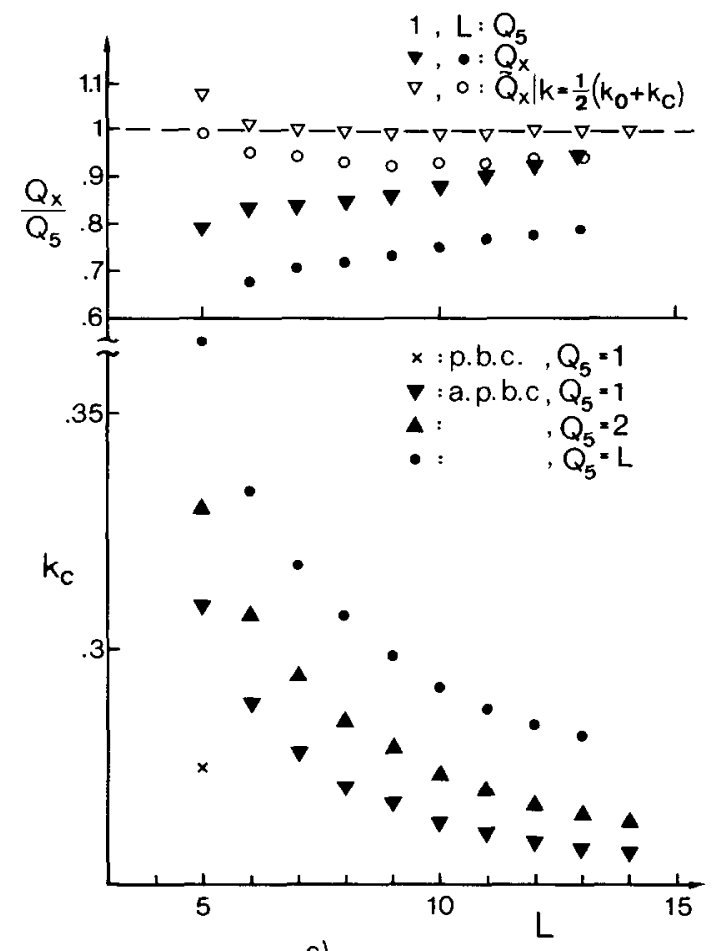

c)

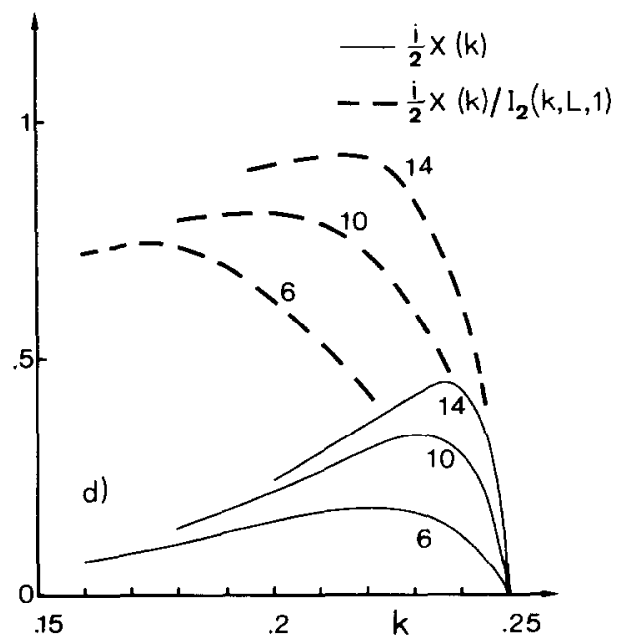

Fig. 4. (continued). 


\section{Topological susceptibility in two-dimensional QED}

In a quantum field theory the quantity of interest it not the topological charge but rather the topological susceptibility. This is so because the total topological charge is something that can be prescribed by imposing boundary conditions and physics should not depend on such an arbitrary prescription. In fact we have to take the thermodynamic limit (infinite volume limit) and since the topological charge density shows local fluctuations with probably exponentially decreasing correlation it does not make sense to talk about the total topological charge in the infinite volume.

On the other hand the topological susceptibility is a quantity related to the decay of correlations between local topological fluctuations and it should be possible to approximate its infinite volume value by a finite volume expression irrespective of boundary conditions.

The topological susceptibility is defined as

$$
\chi=\int \mathrm{d}^{d} x\left(\langle q(x) q(0)\rangle_{\infty}-\lim _{y \rightarrow \infty}\langle q(y) q(0)\rangle_{\infty}\right),
$$

where \langle\rangle$_{\infty}$ is the expectation value in the thermodynamic limit. It is important to take this limit before integrating because otherwise one obtains unwanted boundary dependence as will be shown in some examples below. The second term will not contribute unless there is long range order in the theory.

For numerical studies one has to work with a finite volume, however, and the question is how to approximate (5.1) in a way that is as insensitive as possible to boundary conditions. Boundary conditions in many cases only affect the zero momentum part of the two-point function, i.e. different boundary conditions will manifest themselves mostly by shifting $G(x, y) \equiv\langle q(x) q(y)\rangle$ by a constant.

It is natural to take as a finite volume approximation of (5.1) not

$$
\int_{V} \mathrm{~d}^{d} x\langle q(x) q(0)\rangle=\left\langle Q^{2}\right\rangle / V
$$

as it is usually done [23] but rather

$$
\int_{V} \mathrm{~d}^{d} x\left(\langle q(x) q(0)\rangle-c_{V}\right),
$$

where $c_{V}$ is the "tail value" of $G(x, y)=\langle q(x) q(y)\rangle$ for maximal separation. By this one can hope to eliminate most of the unwanted dependence on boundary conditions.

To see that this is not just idle talk let us look at the simplest of all examples, noncompact $\mathrm{QED}_{2}$ without matter. There we have

$$
q(x)=F(x) \equiv F_{12}(x)
$$


and on a $L \times L$ lattice

$$
\left\langle q_{n} q_{0}\right\rangle=\frac{1}{Z_{L, N}} \int \prod_{i=1}^{L^{2}} \mathrm{~d} F_{i} \times F_{n} F_{0} \exp \left(-\frac{1}{2 g^{2}} \sum_{i} F_{i}^{2}\right) \delta\left(\sum_{i=1}^{L^{2}} F_{i}-N\right),
$$

which gives

$$
\left\langle q_{N} q_{0}\right\rangle_{L, N}=q^{2} \delta_{n 0}-\frac{g^{2}}{L^{2}}+\frac{N^{2}}{L^{4}} .
$$

The topological charge $N$ is fixed by the boundary conditions. Taking $L \rightarrow \infty$ first in (5.6) and then summing over $n$ gives the correct result

$$
\chi=g^{2},
$$

while the formula (5.2) gives, if we first sum over $N$

$$
\chi_{N}=N^{2} / L^{2},
$$

which is not sensible and does not improve much if we take $L \rightarrow \infty$ (at least it becomes independent of $N$ ).

Of course one can improve this situation by averaging over the boundary condition $N$ with a suitably probability measure. Free boundary conditions correspond to the probability

$$
P_{N}=\frac{\exp \left(-N^{2} / 2 g^{2} L^{2}\right)}{\sum_{N} \exp \left(-N^{2} / 2 g^{2} L^{2}\right)}
$$

and therefore free boundary conditions give

$$
\chi_{\mathrm{f}}=\frac{\sum N^{2} p_{N}}{L^{2}} \cong \begin{cases}g^{2} & \text { for } g L \text { large } \\ \frac{1}{L^{2}} & \text { for } g L \text { small }\end{cases}
$$

So this prescription gives at least the right answer in the thermodynamic limit and a reasonable approximation for large volumes.

But it should be stressed again that it does not work at all for some other boundary conditions. Periodic boundary conditions, for instance, correspond to $N=\mathbf{0}$ and give

$$
\chi_{\mathrm{P}}=0 .
$$

Our proposal (5.3), on the other hand, works perfectly for all these boundary 

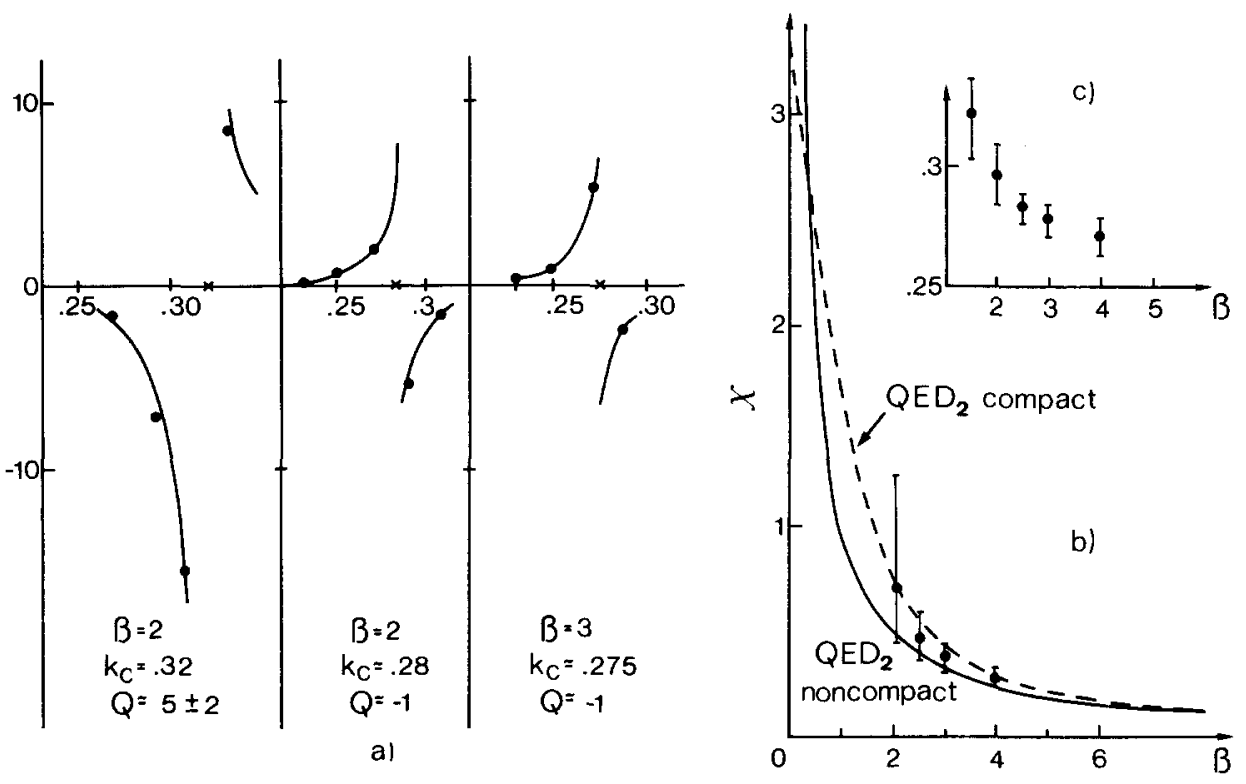

Fig. 5. Topological properties of pure, compact $\mathrm{QED}_{2}$. (a) Fits to $J_{5}(k)$ typical for configurations with nontrivial topology (the value of $\hat{Q}_{5}$ obtained is given on the figure). (b) Topological susceptibility versus $\beta=1 / g^{2}$. (c) $k_{\mathrm{c}}$ as obtained from the fits, averaged at each $\beta$.

conditions. It gives

$$
\chi=g^{2}
$$

already in a finite volume.

Compact $\mathrm{QED}_{2}$ without matter is already a little less trivial. We did a numerical computation of the topological susceptibility $\chi$ using (5.2) and the recipe for extracting $Q_{5}$ described in the previous section. We also measured it by the prescription corresponding to (5.3), i.e.

$$
\chi=\frac{1}{4} \int \mathrm{d}^{2} x(\langle X(x) X(0)\rangle-“ \text { tail" }) .
$$

In this case the tail value turned out to be small compared with the errors introduced by our extrapolation procedures and the results based on the prescriptions (5.2) and (5.3) agree within errors.

In fig. $5 b$ we show our results for the susceptibility of compact $\mathrm{QED}_{2}$ without matter.

The procedure based on (5.2) required first measuring $X$ on each configuration as a function of $k$ and fitting it with the formula (4.9), thereby extracting $\tilde{Q}_{5}$. Typical fits are shown in fig. 5a. In fig. $5 \mathrm{c}$ we show the fit parameters averaged over 
configurations at given $g^{2}$. The lattice we used was $7 \times 7$. We also measured, as remarked, $\chi$ directly using (5.13) and found agreement within errors. The errors are small for small $g^{2}$ where the most probable configurations are quite smooth and have small $Q_{5}$. We used antiperiodic boundary conditions. Because the scaling law here is trivial ( $g=e a, e$ fixed) these results are, however, only of illustrative interest.

\section{Discussion}

Our definition of the lattice topological charge and susceptibility certainly poses very subtle problems, due to the fact that a finite value has to be extracted by letting a pole merge with a zero in $k$. Nevertheless it is possible to extract some sensible numbers.

The subtleties are, however, to a large extent contained in the normalization factor $I(d)$ which has indeed a tricky behavior that can lead easily to confusion (see for instance [24]).

We have shown that the assumed analyticity structure of $X(k)$ seems to be correct and that the ansatz based on it allows to extract the topological charge of fixed gauge configurations in a reliable way, thereby giving some a posteriori justification to this ansatz.

In general it is difficult to extract the topological susceptibility, which is the quantity of interest, from our definition. But we managed to do so for $\mathrm{QED}_{2}$.

Our method of defining and measuring topological charge and topological susceptibility is certainly rather complicated compared to the "interpolation method" proposed by Lüscher [25] and related definitions [26]. But it has one property not shared by other definitions [12]: it preserves the important link between topological properties of the gauge field and chiral properties of the fermions that is expressed in the continuum by the index theorem. Therefore it would be quite worthwhile to try to extract a "physical value" of the topological susceptibility from a realistic simulation of $\mathrm{QCD}_{4}$ using our method.

E.S. would like to thank E. Lieb and A.S. Wightman for their kind hospitality at the Princeton Physics Department. I.O.S. would like to thank H. Meyer-Ortmanns, Jan Smith and K. Yoshida for many interesting discussions and information on their results. F.K. acknowledges partial support from the National Science Foundation under the grant PHY82-01948.

\section{References}

[1] M.F. Atiyah and I.M. Singer, Bull. Am. Math. Soc. 69 (1963) 422; Ann. of Math. 87 (1968) 546; P. Gilkey, The Atiyah-Singer index theorems and the heat equation (Publish or Perish, Boston, 1974);

E. Getzler, Comm. Math. Phys. 92 (1983) 163; Harvard preprint HUTMP B 156 (1984);

T. Eguchi, P. Gilkey and A. Hanson, Phys. Reports 66 (1980) 213 
[2] M.F. Atiyah and I.M. Singer, Ann. of Math. 93 (1971) 119;

M.F. Atiyah, R. Bott and V.K. Patodi, Ann. of Math. 19 (1973) 279;

L. Alvarez-Gaumé, Comm. Math. Phys. 90 (1983) 161;

C. Callias, Comm. Math. Phys. 62 (1978) 213;

R. Bott and R. Seeley, Comm. Math. Phys. 62 (1978) 235;

A.J. Niemi and G.W. Semenoff, Institute for Advanced Study (Princeton) preprint (1985)

[3] N.K. Nielsen and B. Schroer, Nucl. Phys. B127 (1977) 493;

N.K. Nielsen, H. Römer and B. Schroer, Phys. Lett. 70B (1977) 445;

Nucl. Phys. B136 (1978) 475

[4] E. Witten, Nucl. Phys. B156 (1979) 269;

G. Veneziano, Nucl. Phys. B159 (1979) 213

[5] G. 't Hooft, Phys. Rev. Lett. 37 (1976) 8

[6] J. Schwinger, Phys. Rev. 82 (1951) 664;

J.S. Bell and R. Jackiw, Nuovo Cim. 60A (1969) 47;

S. Adler, Phys. Rev. 177 (1969) 2426;

W.A. Bardeen, Phys. Rev. 184 (1969) 1848

[7] K. Fujikawa, Phys. Rev. Lett. 42 (1979) 1195, 44 (1980) 1733; Phys. Rev. D21 (1980) 2848, D22 (1980) 1499

[8] A. Andrianov and L. Bonora, Nucl. Phys. B233 (1984) 232, 247

[9] K.G. Wilson, Phys. Rev. D10 (1974) 2445; in New phenomena in subnuclear physics, ed. A. Zichichi (Plenum, New York, 1976)

[10] E. Seiler and I.O. Stamatescu, Phys. Rev. D25 (1982) 2177

[11] G. Immirzi and K. Yoshida, Nucl. Phys. B210 [FS6] (1982) 499

[12] M. Teper, Proc. Conf. on Quark confinement and liberation: numerical results and theory (Berkeley, 1985) to be published

[13] F.A. Berezin, The method of second quantization (Academic Press, New York, 1966)

[14] L.H. Karsten and J. Smit, Nucl. Phys. B183 (1981) 103

[15] L. Susskind, Phys. Rev. D16 (1977) 3031;

H.S. Sharatchandra, H.J. Thun and P. Weisz, Nucl. Phys. B192 (1981) 205;

P. Becher, Phys. Lett. B104 (1981) 221;

E. Kähler, Rand. Mat. (Roma) Ser. V 21 (1962) 425

[16] W. Kerler, Phys. Rev. D23 (1981) 2384

[17] N. Kawamoto, Nucl. Phys. B190 [FS3] (1981) 617

[18] J. Hoek, J. Smit and N. Kawamoto, Nucl. Phys. B199 (1982) 495

[19] I.O. Stamatescu, Quarks, leptons and beyond, NATO ASI Series (Plenum, 1985)

[20] M. Bochicchio, preprint 416 (Roma, 1984)

[21] I.O. Stamatescu, Phys. Rev. D25 (1981) 1130

[22] M. Peskin, thesis, Cornell Univ. preprint CLNS 395 (1978)

[23] P. Di Vecchia, K. Fabricius, G.C. Rossi and G. Veneziano, Nucl. Phys. B192 (1981) 392

[24] N. Kawamoto and K. Shigemoto, Phys. Lett. 120B (1983) 183

[25] M. Lüscher, Comm. Math. Phys. 85 (1982) 39

[26] P. Woit, Phys. Rev. Lett. 51 (1983) 638 